\title{
Influence of Pressure on the Electrical Transport Properties of Carbon-Doped $\mathrm{EuB}_{6}$
}

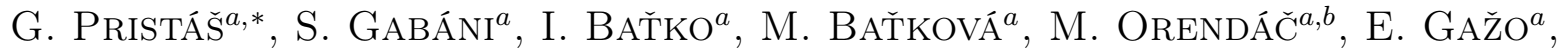 \\ V. Filipov ${ }^{c}$, E. KonOVAlOVA ${ }^{c}$, P. DiKo ${ }^{a}$ AND K. FlachBART ${ }^{a}$ \\ ${ }^{a}$ Institute of Experimental Physics, Slovak Academy of Sciences, Watsonova 47, 04001 Košice, Slovakia \\ ${ }^{b}$ Institute of Physics, Faculty of Sciences, P.J. Safarik University, Park Angelinum 9, 04001 Košice, Slovakia \\ ${ }^{c}$ Institute for Problems of Material Science, National Academy of Sciences of Ukraine, \\ Krzhyzhanovsky 3, 03680 Kiev, Ukraine
}

\begin{abstract}
We have studied the influence of hydrostatic pressure on the electrical resistivity of carbon-doped semimetal $\mathrm{EuB}_{6}$ which orders ferromagnetically at $T_{\mathrm{C}}=3.9 \mathrm{~K}$ and is intrinsically inhomogeneous due to fluctuations of carbon content. We observed a shift of the low-temperature resistivity maximum from $4.6 \mathrm{~K}$ (at 1 bar) to $5.2 \mathrm{~K}$ (at 30.3 kbar) with increasing pressure. However, the maximum of the derivative $\mathrm{d} \rho / \mathrm{d} T(T)$, which reveals the temperature of ferromagnetic ordering, does not change its position with increasing pressure. This behaviour is different from stoichiometric $\mathrm{EuB}_{6}$, where pressure increases the ferromagnetic ordering temperature. The origin of this discrepancy may lie in the increase of volume fraction of the non-ferromagnetic phase with increase of pressure. Additional magnetoresistance measurements at various pressures between $1.5 \mathrm{~K}$ and $30 \mathrm{~K}$ have shown that with increase of magnetic field the resistivity is monotonically decreasing, and above $1 \mathrm{~T}$ a transition to a monotonic resistivity behaviour $(\mathrm{d} \rho / \mathrm{d} T(T)>0)$ is observed. Our results support the picture that carbon-rich regions play a role of "spacers", which prevent the percolation of the ferromagnetic phase.
\end{abstract}

DOI: 10.12693/APhysPolA.131.982

PACS/topics: $74.62 . \mathrm{Fj}, 75.30 . \mathrm{Kz}$

\section{Introduction}

The crystallographic simplicity (simple cubic lattice) of $\mathrm{EuB}_{6}$ makes this system ideal for studying the interplay between electronic and magnetic properties under the influence of doping. In the last few decades the physical properties of $\mathrm{EuB}_{6}$ have been studied in many works (e.g. Refs. [1-4]). High quality single-crystalline samples of $\mathrm{EuB}_{6}$ reveal two ferromagnetic (FM) transitions at $T_{c 1}=15.3 \mathrm{~K}$ and $T_{c 2}=12.5 \mathrm{~K}$ and undergo a metalinsulator phase transition [1]. As it was shown by highpressure experiments [5], the $\mathrm{FM}$ order in $\mathrm{EuB}_{6}$ is driven by the Ruderman-Kittel-Kasuya-Yosida (RKKY) interaction. Due to a very low number of intrinsic charge carriers $\left(\approx 10^{20} \mathrm{~cm}^{-3}\right)$, even small doping with carbon can drastically modify its electric and magnetic properties [6-8].

In presented work, we have studied the influence of hydrostatic pressure on carbon-doped $\mathrm{EuB}_{6}$ which exhibits a transition temperature to $\mathrm{FM}$ state at $T_{\mathrm{C}}=3.9 \mathrm{~K}$. Carbon-doped $\mathrm{EuB}_{6}$ is intrinsically inhomogeneous due to fluctuations of carbon content [8]. The main differences between $\mathrm{EuB}_{6}$ and carbon-doped $\mathrm{EuB}_{6}$ are as follows. (i) The paramagnetic state of $\mathrm{EuB}_{6}$ is homogeneous, however, the paramagnetic state in carbondoped $\mathrm{EuB}_{6}$ is inhomogeneous, containing regions with increased carbon content (having a higher electrical conductance than the remaining matrix). (ii) The magnetic

*corresponding author; e-mail: gabriel.pristas@saske.sk polaron (MP) phase (below about $30 \mathrm{~K}$ [4]) in $\mathrm{EuB}_{6}$ has two components: a highly-conducting FM phase (represented by MPs) and a poorly conducting paramagnetic phase. In case of carbon-doped $\mathrm{EuB}_{6}$ this phase consists at least of three components [9]: a highly-conducting FM phase represented by MPs (matrix), and regions with carbon-poor and carbon-rich domains. (iii) In the magnetically ordered state $\mathrm{EuB}_{6}$ is a homogeneous ferromagnet, while carbon-doped $\mathrm{EuB}_{6}$ has two components: the FM matrix and heli-magnetic (HM) domains formed in carbon-rich regions [8]. In this work we present the influence of pressure on the resistivity and magnetoresistivity of carbon-doped $\mathrm{EuB}_{6}$.

\section{Experimental details}

The $\mathrm{EuB}_{6-x} \mathrm{C}_{x}$ single crystal was grown using the zone-floating method and we used a sample from the same batch as in work [9], where the content of carbon was estimated by microprobe to be $x=0.01$. Measurements of ac-resistivity have been performed by means of a four probe method utilizing lock-in amplifier and current source with active common mode rejection. Contacts were made using silver paste and gold wires. In case of magnetoresistance measurements the direction of the applied magnetic field was perpendicular to electrical current. Hydrostatic pressure was generated in a pistoncylinder pressure cell with pressure medium - Daphne 7373 oil. For determining the pressure we used the superconducting transition of lead. Measurements were performed in the temperature range between $1.6 \mathrm{~K}$ and $300 \mathrm{~K}$ using a ${ }^{4} \mathrm{He}$ flow cryostat. 


\section{Results and discussion}

In Fig. 1 we show the temperature dependence of resistivity in temperature range between 1.6 and $30 \mathrm{~K}$, to emphasize the influence of pressure on the low temperature part of resistivity. As can be seen, the maximum of resistivity shifts monotonically to higher temperature and its absolute value is decreasing with increase of pressure. An interesting feature is also the non-monotonic dependence of the absolute resistivity value below $T_{\mathrm{C}}$ (e.g. at $2 \mathrm{~K}\left(\rho_{2} \mathrm{~K}\right)$, see inset in Fig. 1) which points to an interplay between at least two mechanisms - one is suppressing and the other one increasing the resistivity with pressure. On the other hand, the monotonic decrease of resistivity at room temperature $\left(\rho_{300 \mathrm{~K}}\right.$, not shown $)$ by $7 \%$, from $163 \mu \Omega \mathrm{cm}$ to $151.7 \mu \Omega \mathrm{cm}$, can be explained by an increase of the concentration of charge carriers due to applied pressure [9]. In case of stoichiometric $\mathrm{EuB}_{6}$ a decrease of $\rho_{300 \mathrm{~K}}$ by about $20 \%$ was observed in the same pressure range [5]. This decrease is connected with the closing of the energy gap $E_{g}[5]$. Thus, the effect of pressure at low temperatures seems to be more complicated.

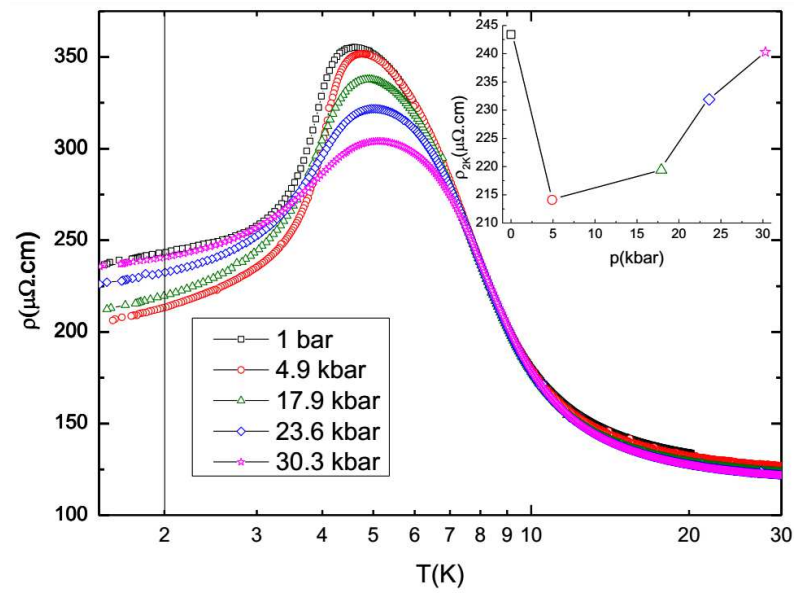

Fig. 1. Temperature dependence of resistivity of carbon-doped $\mathrm{EuB}_{6}$ at different applied pressures. In inset the change of the absolute value of resistivity at temperature $2 \mathrm{~K}\left(\rho_{2} \mathrm{~K}\right)$ with increase of pressure is shown (lines between points are to guide the eye).

In order to see the pressure influence on the transition temperature to the FM state in the carbon doped sample, we made the numerical derivative of $\rho(T)$. The Curie temperature $T_{\mathrm{C}}$ was determined from the derivative maxima of resistivity as in metallic ferromagnets [10]. As one can see in Fig. 2, the maximum of $\mathrm{d} \rho / \mathrm{d} T$ does not change its position with pressure within experimental error. This result supports the picture that carbon-rich regions play a role of "spacers", which prevent the percolation of the FM phase. On the other, hand in case of stoichiometric $\mathrm{EuB}_{6}$, the influence of pressure is much more pronounced. There the increase of $T_{\mathrm{C}}$ with pressure is about $30 \%$ up to $50 \mathrm{kbar}$ and at higher pressure it has a tendency to saturate [5]. Therefore, we suppose that in doped $\mathrm{EuB}_{6}$ the increase of pressure increases the volume of the non-FM phase (HM domains) formed in carbon-rich regions and this prevents the percolation of the FM phase. But, because the temperature of FM ordering of the carbon-poor matrix increases with pressure (as in pure $\mathrm{EuB}_{6}$ ), as a result we have "no shift" of bulk $T_{\mathrm{C}}$. The cutting points of derivatives with $\mathrm{d} \rho / \mathrm{d} T=0$ correspond in Fig. 2 to $\rho(T)$ maxima and the extracted pressure dependence of $T_{R M}$ (temperature of resistivity maximum) is shown in the inset of Fig. 2. With increasing pressure we see an increase of $T_{R M}$ which is opposite to the dependence observed in our previous work [9]. However, the result reported in this work is consistent with the influence of pressure on pure $\mathrm{EuB}_{6}$. As it was already shown in previous studies [1-9], the "sample dependence issue" is important in case of $\mathrm{EuB}_{6}$ and carbon-doped $\mathrm{EuB}_{6}$. The reason for it is the very small number of intrinsic carriers, which influences considerably the physical properties of samples, and even very small deviations in stoichiometry or impurities can lead to a substantially different behaviour. Therefore, one reason for the discrepancy between the observed $T_{R M}$ behaviours can be a small deviation of the carbon concentration in spite of that we used samples from the same batch. Comparing the value of maximum in temperature dependence of resistivity with previous works $[11,12]$, we estimated the concentration of carbon to be around $x=0.03$.

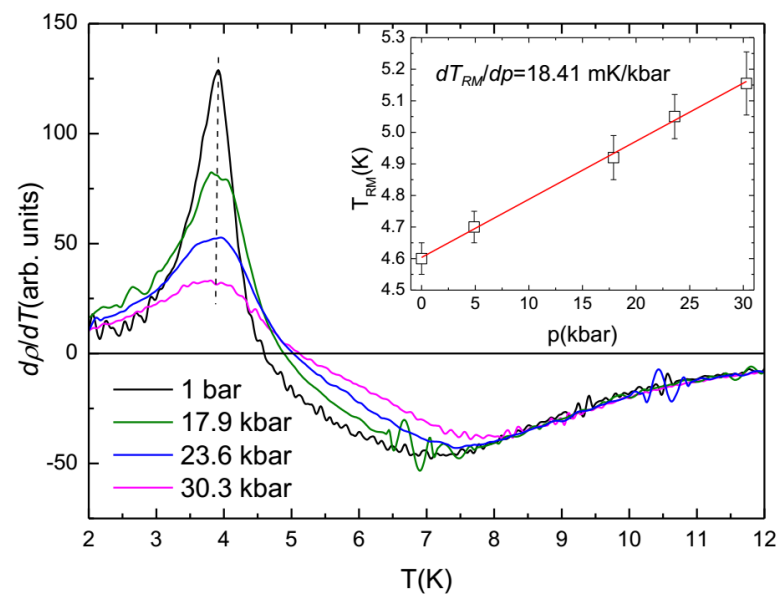

Fig. 2. Derivatives of resistivity data $\mathrm{d} \rho / \mathrm{d} T(T)$ at different pressures. The inset shows the shift of resistivity maximum with increasing pressure (the red line is the linear fit of data).

Figures 3 and 4 show the temperature dependences of resistivity of carbon-doped $\mathrm{EuB}_{6}$ in various magnetic fields under pressures of 4.9 and 30.3 kbar. In case of 4.9 kbar we observed a suppression of resistivity with increasing magnetic field and the maximum at $4.7 \mathrm{~K}$ moves to lower temperatures. In field of $2 \mathrm{~T}$ we have a monotonic resistivity behaviour $(\mathrm{d} \rho / \mathrm{d} T(T)>0)$. At higher pressure $(30.3 \mathrm{kbar})$ the applied field above $1 \mathrm{~T}$ splits the $\rho(T)$ maximum. This behaviour points to a delicate interplay between all above mentioned magnetic components in carbon-doped $\mathrm{EuB}_{6}$. 


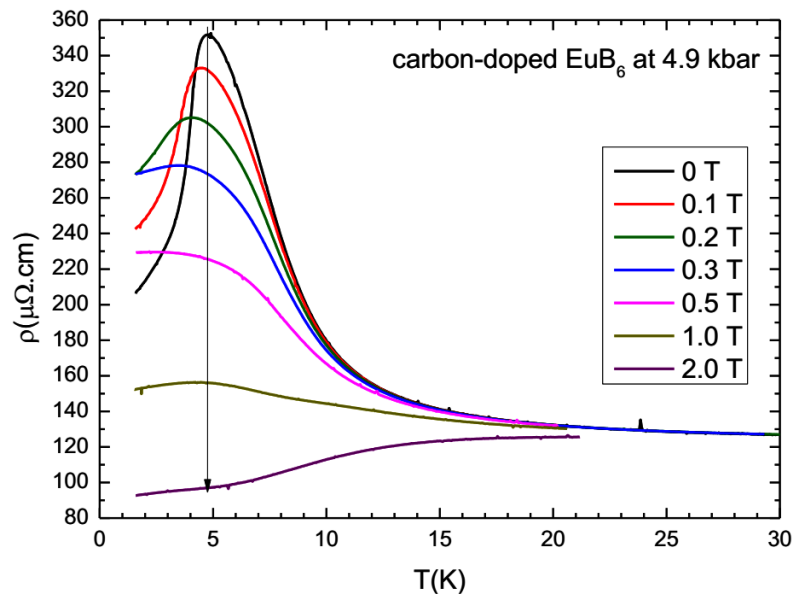

Fig. 3. Temperature dependences of resistivity of carbon-doped $\mathrm{EuB}_{6}$ in various magnetic fields at 4.9 kbar.

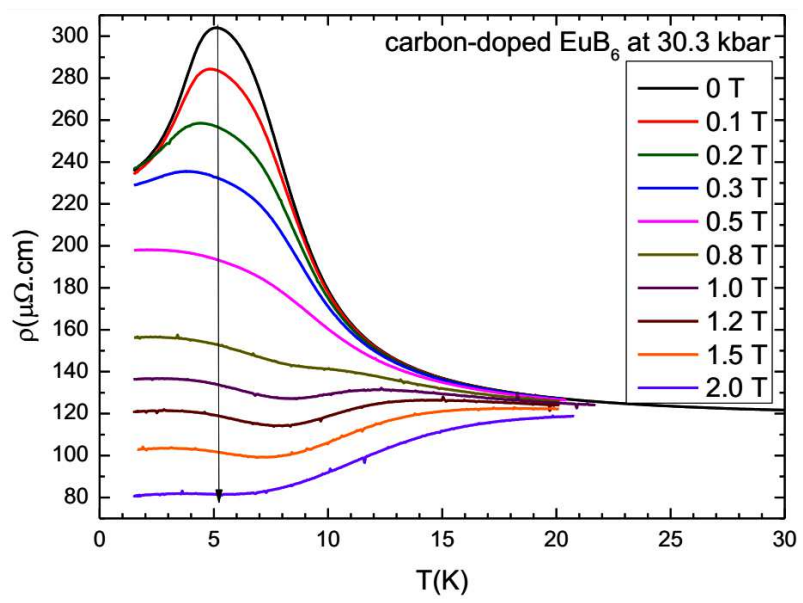

Fig. 4. Temperature dependences of resistivity of carbon-doped $\mathrm{EuB}_{6}$ in various magnetic fields at 30.3 kbar.

\section{Conclusions}

We have studied the influence of pressure up to $30.3 \mathrm{kbar}$ on transport properties of carbon-doped $\mathrm{EuB}_{6}$. No shift of the ordering temperature to the FM state with pressure was observed, which is consistent with our previous work performed on similar sample up to $10.3 \mathrm{kbar}$ [9]. However, in the present work we have observed a different behaviour of the pressure dependence of the $\rho(T)$ maximum. This discrepancy may be attributed to the slightly different concentration of carbon (estimated to be around $x=0.03$ ), and to the very high sensitivity of sample properties to the carbon content. The not changing $T_{\mathrm{C}}$ with pressure points to fact that the HM phase, which is connected with carbon-rich regions, is suppressing the value of $T_{\mathrm{C}}$ with increasing pressure, and that the phase connected with MP is in the same extent increasing the value of $T_{\mathrm{C}}$ (as in pure $\mathrm{EuB}_{6}$ ). To solve this problem in detail, a careful carbon distribution analysis of investigated samples will be needed. Moreover, it will be interesting to apply higher pressure to see which of the above mentioned phases will prevail with the increase of pressure.

\section{Acknowledgments}

This work was supported by project VEGA 2/0032/16, VEGA 2/0184/13, APVV-0605-14, APVV-0330-12 and EU ERDF-ITMS 26220120047.

\section{References}

[1] S. Süllow, I. Prasad, M.C. Aronson, J.L. Sarrao, Z. Fisk, D. Hristova, A.H. Lacerda, M.F. Hundley, A. Vigliante, D. Gibbs, Phys. Rev. B 57, 5860 (1998).

[2] Z. Fisk, D.C. Johnston, B. Cornut, S. von Molnar, S. Oseroff, R. Calvo, J. Appl. Phys. 50, 133 (1979).

[3] L. Deriorgi, E. Felder, H.R. Ott, J.L. Sarrao, Z. Fisk, Phys. Rev. Lett. 79, 5134 (1997).

[4] S. Süllow, I. Prasad, M.C. Aronson, S. Bogdanovich, J.L. Sarrao, Z. Fisk, Phys. Rev. B 62, 11626 (2000).

[5] J.C. Cooley, M.C. Aronson, J.L. Sarrao, Z. Fisk, Phys. Rev. B 56, 14541 (1997).

[6] M.C. Aronson, J.L. Sarrao, Z. Fisk, M. Whitton, B.L. Brandt, Phys. Rev. B 59, 4720 (1999).

[7] M. Batkova, I. Batko, K. Flachbart, Z. Janu, K. Jurek, E.S. Konovalova, J. Kováč, M. Reiffers, V. Sechovský, N. Shitsevalova, E. Šantavá, J. Šebek, Phys. Rev. B 78, 224414 (2008).

[8] J.M. Tarascon, J.L. Soubeyroux, J. Etourneau, R. Georges, J.M.D. Coey, O. Massenet, Solid State Commun. 37, 133 (1981).

[9] M. Batkova, I. Batko, E. Bauer, R.T. Khan, V.B. Filipov, E.S. Konovalova, Solid State Commun. 150, $652(2010)$.

[10] M.E. Fisher, J.S. Langer, Phys. Rev. Lett. 20, 665 (1968).

[11] S. von Molnar, J.M. Tarascon, J. Etourneau, J. Appl. Phys. 50, 2158 (1981).

[12] T. Fujita, M. Suzuki, Y. Isikawa, Solid State Commun. 33, 947 (1980). 